\title{
Illustrated key to the adult female Anopheles (Diptera: Culicidae) mosquitoes of Sri Lanka
}

\author{
Nayana Gunathilaka ${ }^{1}$
}

Received: 11 August 2016 / Accepted: 20 September 2016 / Published online: 28 December 2016

(C) The Author(s) 2016. This article is published with open access at Springerlink.com

\begin{abstract}
The identification of adult female anopheline mosquitoes is an important aspect in malaria surveillance and control strategy throughout the world, and taxonomic keys are being regularly revised and updated as new information becomes available. However, the currently available key to the anophelines of Sri Lanka is of limited use, because they were published more than 25 years ago. This paper presents an illustrated key for the identification of 23 adult female Anopheles mosquitoes which are currently recognized as local anopheline species in Sri Lanka.
\end{abstract}

Keywords Anopheles · Vector

\section{Introduction}

The genus Anopheles is the only mosquito taxon known to transmit human malarial protozoa. Anophelines are also known to be capable of transmitting dirofilarial nematodes and arboviruses of veterinary and medical importance (Ramachandra 1984). The taxonomy of this group of mosquitoes is, therefore, of great importance, since vector incrimination is dependent upon accurate species identification. Traditional field taxonomy based on morphological characteristics remains the backbone of all vector control programs. Therefore, there is a need for continual revision and improvement of morphological keys for the identification of members of this important group of insects (Amarasinghe 1990).

Nayana Gunathilaka

n.gunathilaka@kln.ac.lk

1 Department of Parasitology, Faculty of Medicine, University of Kelaniya, Ragama, Sri Lanka
Previous records of Sri Lankan Anopheles were imperfect, and even the number and names of the species present were doubtful. An attempt to remedy the matter was made by Dr. A. J. Chalmers in 1905. Chalmers presented the results of investigations made in various parts of the island during the dry season, and also incorporated the records of previous observers. The species list was revised by Carter (1950) and subsequently by Jayasekara and Chelliah (1981). Twenty-two anopheline species were recorded in Sri Lanka by Amarasinghe (1990).

Two illustrated keys to the Anopheles of Sri Lanka (Amarasinghe 1990; Carter 1950) are of limited value, as these were published more than 25 years ago and significant advances in our knowledge of the Anopheles mosquitoes have occurred in the intervening years. The purpose of the key presented herein is to assist entomologists in identifying adult female Anopheles mosquitoes. The key can be used to initially allocate specimens to species group and then to species.

\section{Materials and methods}

The morphological characteristics used here were based on original observations and previous usage in the literature. The following publications were consulted during the construction of this key (Amarasinghe 1990; Christophers 1933; Colless 1957; Harrison 1980; Harrison and Scanlon 1975; Reid 1968; Rattanarithikul et al. 2006; Sallum et al. 2005).

Taxonomic characteristics were checked against Sri Lankan specimens by examining the persevered reference specimens archived at the Faculty of Medicine, University of Kelaniya, Sri Lanka.

Generally, two or more primary characteristics are used in each step in the key, with the intension of making them user-friendly for field taxonomists. Species 


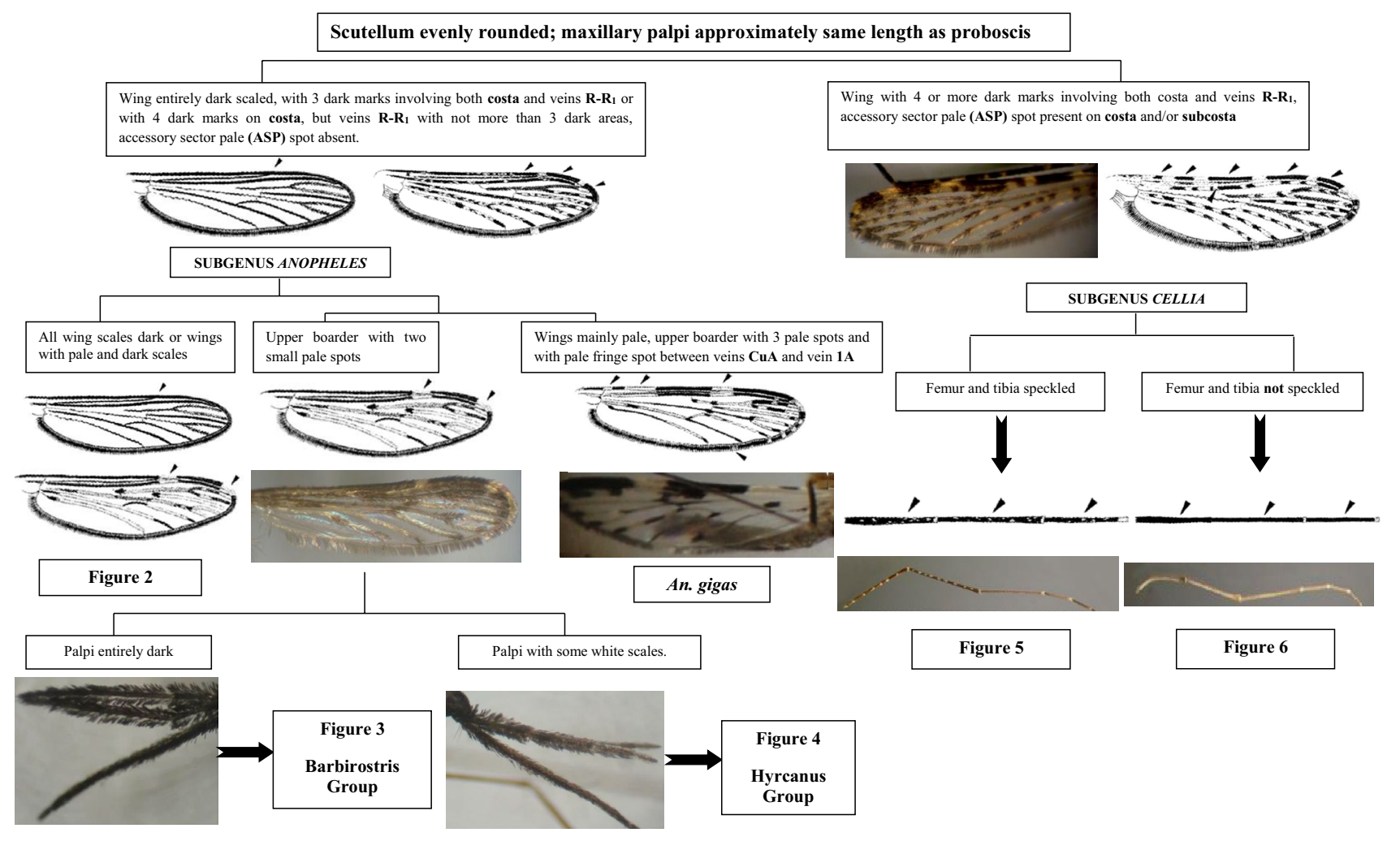

Fig. 1 Key to the adult female anophelines in Sri Lanka

nomenclature follows that proposed by Knight and Stone (1977), and abbreviations used in the text follow those used by Reinert (1975, 2001). Nomenclature for morphological characteristics follows Harbach and Knight (1980, 1982).

The key was distributed to the 17 field entomological surveillance teams in Mannar, Trincomalee, Ampara, Batticaloa, and Killinochchi Districts attached to the Tropical and Environmental Diseases and Health Associates (TEDHA) malaria elimination program and to some selected entomological teams in the Anti Malaria Campaign (AMC).

Reported species were identified using the key for an 8-month period, and feedback was obtained from the field entomological teams. The key was validated on the basis of the feedback. In case of doubt, it is essential to consult published literature with detailed descriptions of species.

\section{Results}

This section presents an illustrated key for the identification of 23 adult female Anopheles mosquitoes which are currently recognized as the local anopheline species in Sri Lanka. Morphological features of An. peytoni and An. jeyporiensis have been included in addition to the anopheline keys published previously. The revised adult morphological key is shown below (Figs. 1, 2, 3, 4, 5, 6, 7, 8, 9). The species included in the key are as follows:

Subgenus Anopheles: aitkenii James, barbirostris Van der Wulp, barbumbrosus Strickland and Choudhury, gigas refutans Alcock, interruptus Puri, nigerrimus Giles, peditaeniatus (Leicester), peytoni Kulasekera, Harrison and Amerasinghe, reidi Harrison.

Subgenus Cellia: aconitus Donitz, annularis Van der Wulp, culicifacies Giles, elegans (James), jamesii Theobald, jeyporiensis James, karwari (James), maculatus Theobald, pallidus Theobald, pseudojamesi Strickland and Chowdhury, subpictus Grassi, tessellatus Theobald, vagus Donitz, varuna Iyengar.

\section{Discussion}

This key is meant as an aid to the rapid identification of anophelines in Sri Lanka. It has thus been made as simple and concise as possible, using a few recognized primary characteristics at each step. Steps that would assign various species to their respective series and species groups are included wherever essential.

The illustrated key includes 23 Anopheles species which are currently known to occur in Sri Lanka. Anopheles peytoni and An. jeyporiensis, which were not included 


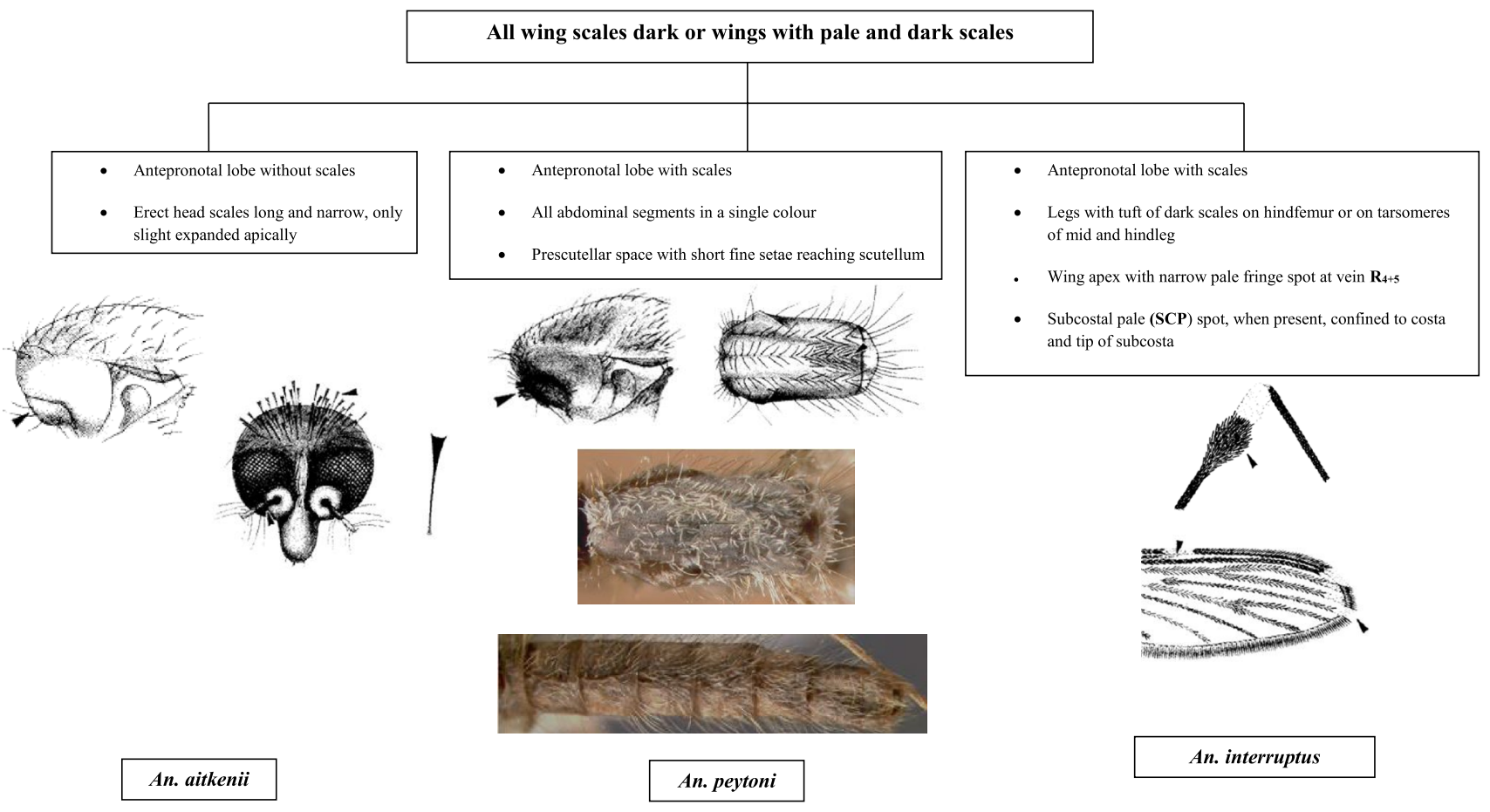

Fig. 2 Mosquitoes under subgenus Anopheles with all wing scales dark or wings with pale and dark scales

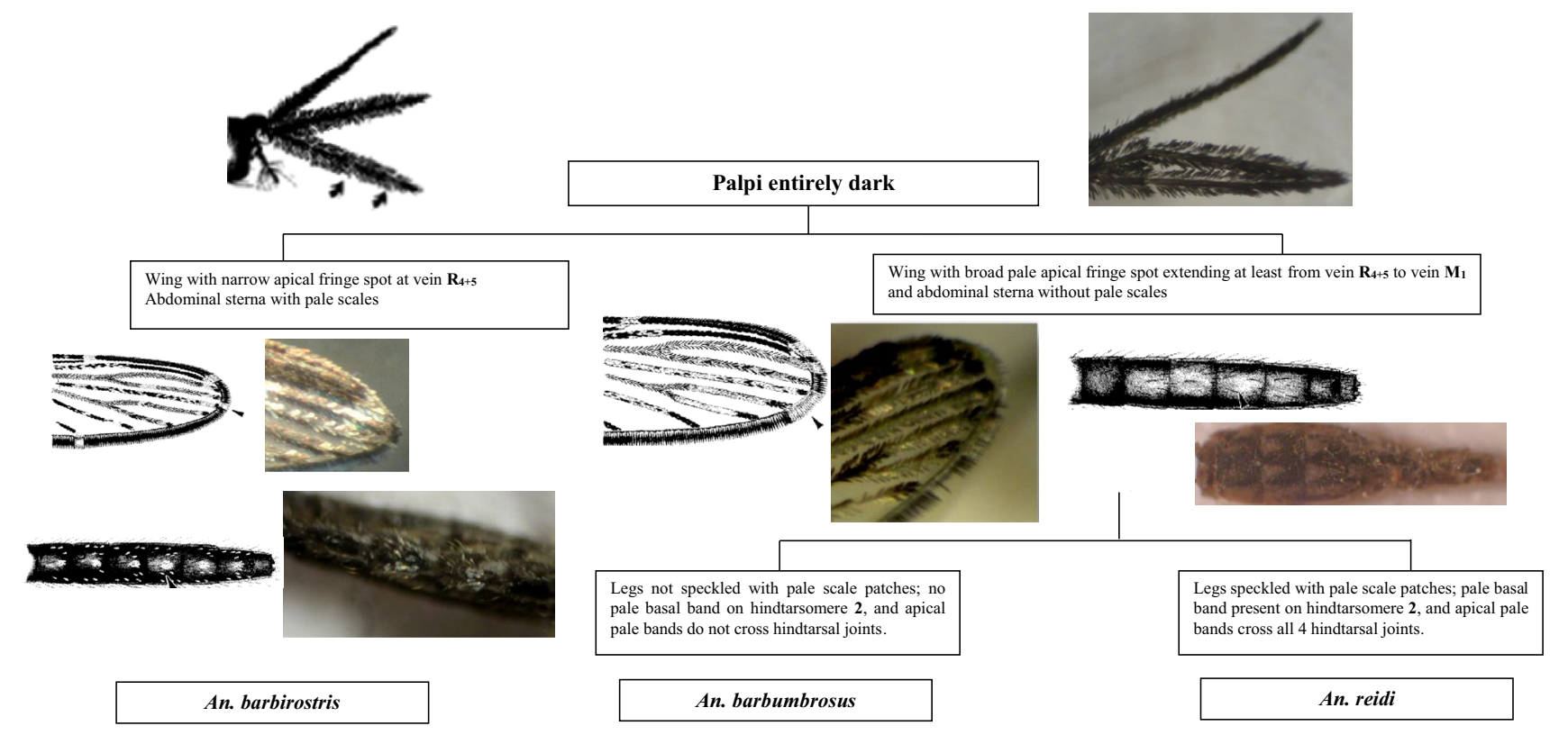

Fig. 3 Members in the Barbirostris group

in previous keys for the anophelines of Sri Lanka, are included here.

Subgenus Anopheles: The following morphological features distinguish species of this subgenus from those of the subgenus Cellia: wings entirely dark-scaled or with three dark marks involving the costa and veins $\mathrm{R}-\mathrm{R}_{1}$. The Sri
Lankan anophelines of this subgenus belong to three taxonomic series: the Myzorhynchus, Lophoscelomyia, and Anopheles series.

Species of the Myzorhynchus series can be separated from species of the other series in Sri Lanka by following characteristics: (i) wing with dark and pale scales, 


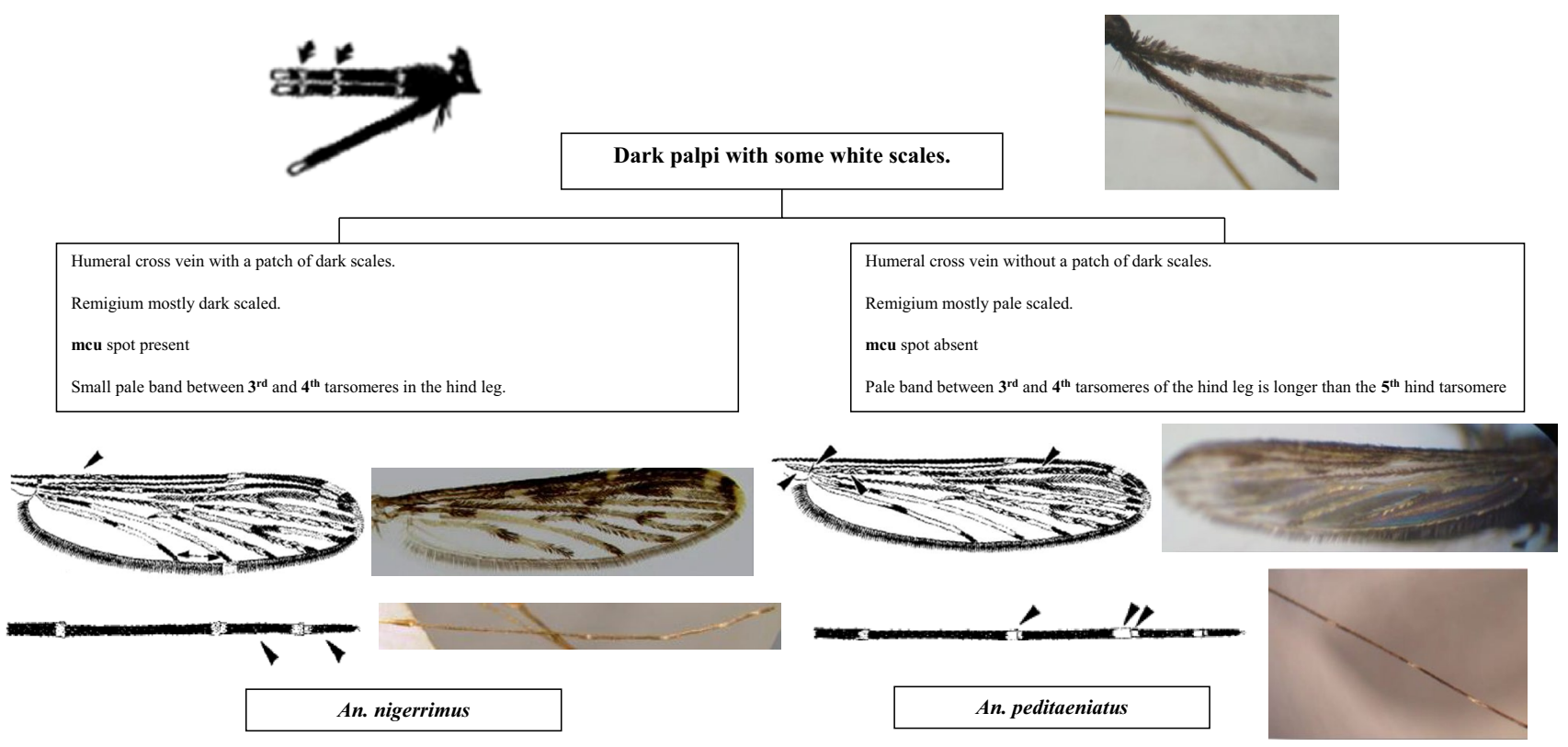

Fig. 4 Members in the Hycanus group

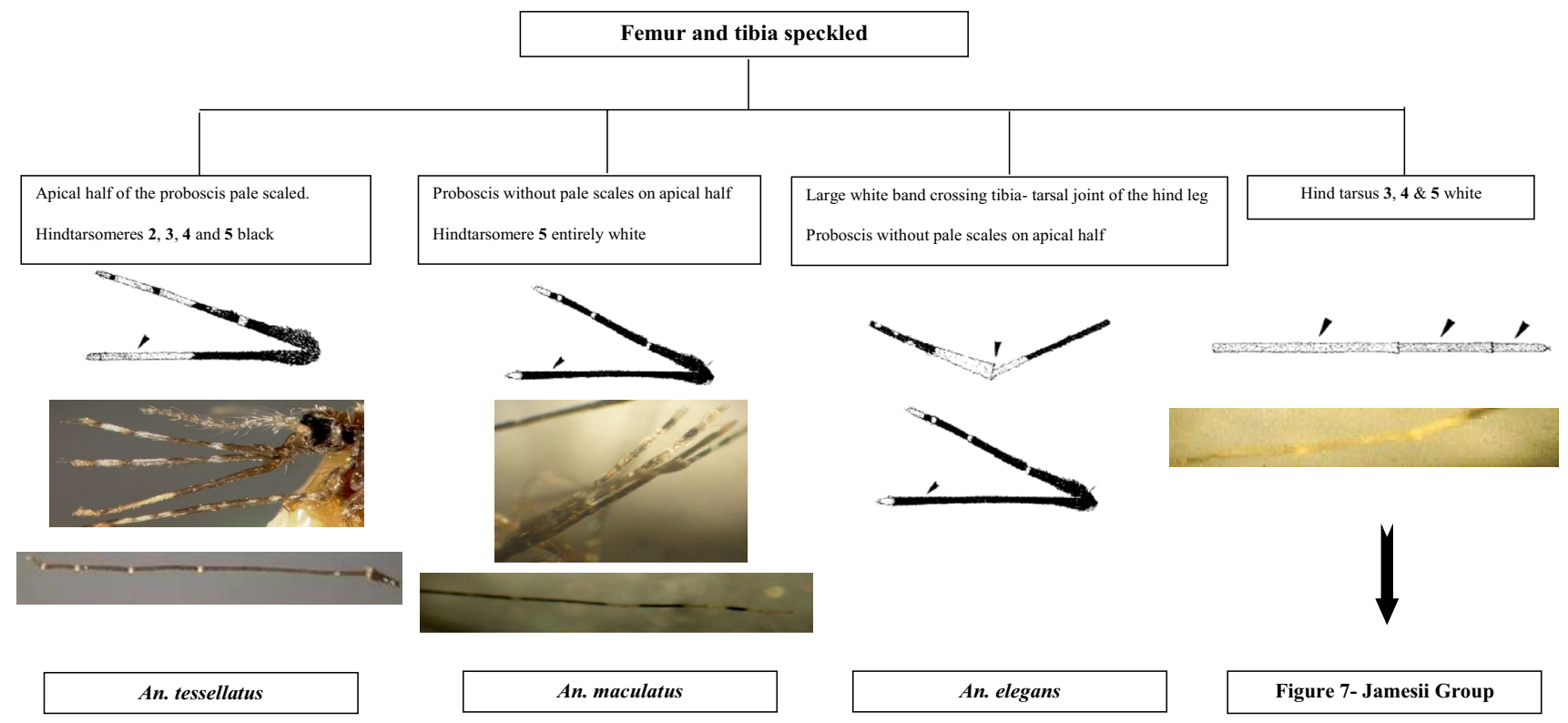

Fig. 5 Members in the Subgenus Cellia with fumur and tibia speckled

upper boarder with two small pale spots; (ii) antepronotum with scales; (iii) maxillary palp with dark and erect scales (shaggy appearance); (iv) basal third of fore femur swollen; (v) hind femur without a distal broad white band; (vi) head scales always broad; (vii) coxae often with scales; and (viii) tarsi with pale bands.

Species of the Hyrcanus and Barbirostris groups of the Myzorhynchus series are present in Sri Lanka. These two groups can be separated easily by the ornamentation of the maxillary palpus, which is entirely dark-scaled in species of the Barbirostris group and has some white scales in species of the Hyrcanus group.

The Hyrcanus group includes two species in Sri Lanka, An. nigerrimus and An. peditaeniatus. There is a considerable degree of confusion regarding their identification. The most reliable identification features are included in the key. 


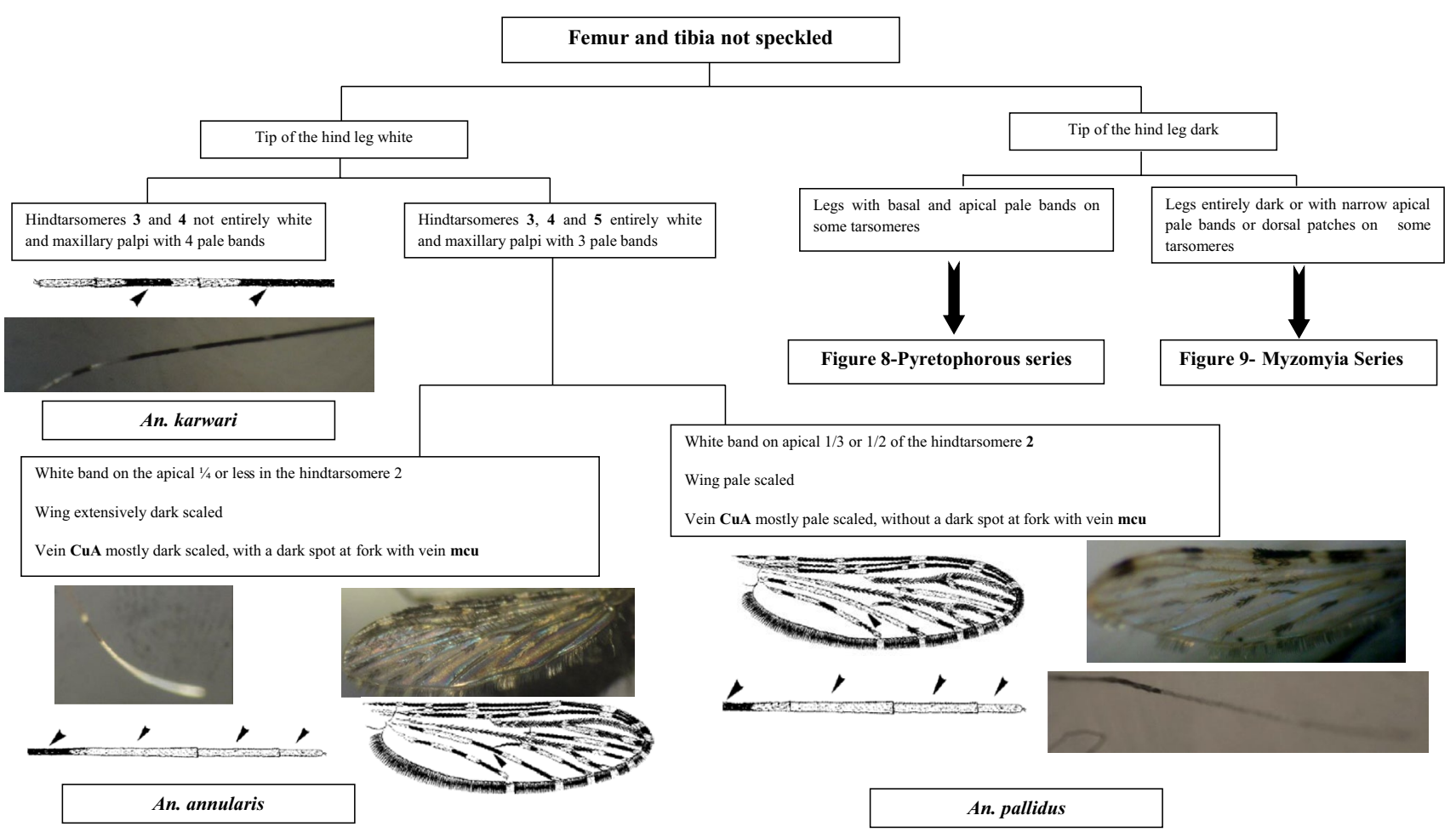

Fig. 6 Members in the Subgenus Cellia with fumur and tibia not speckled

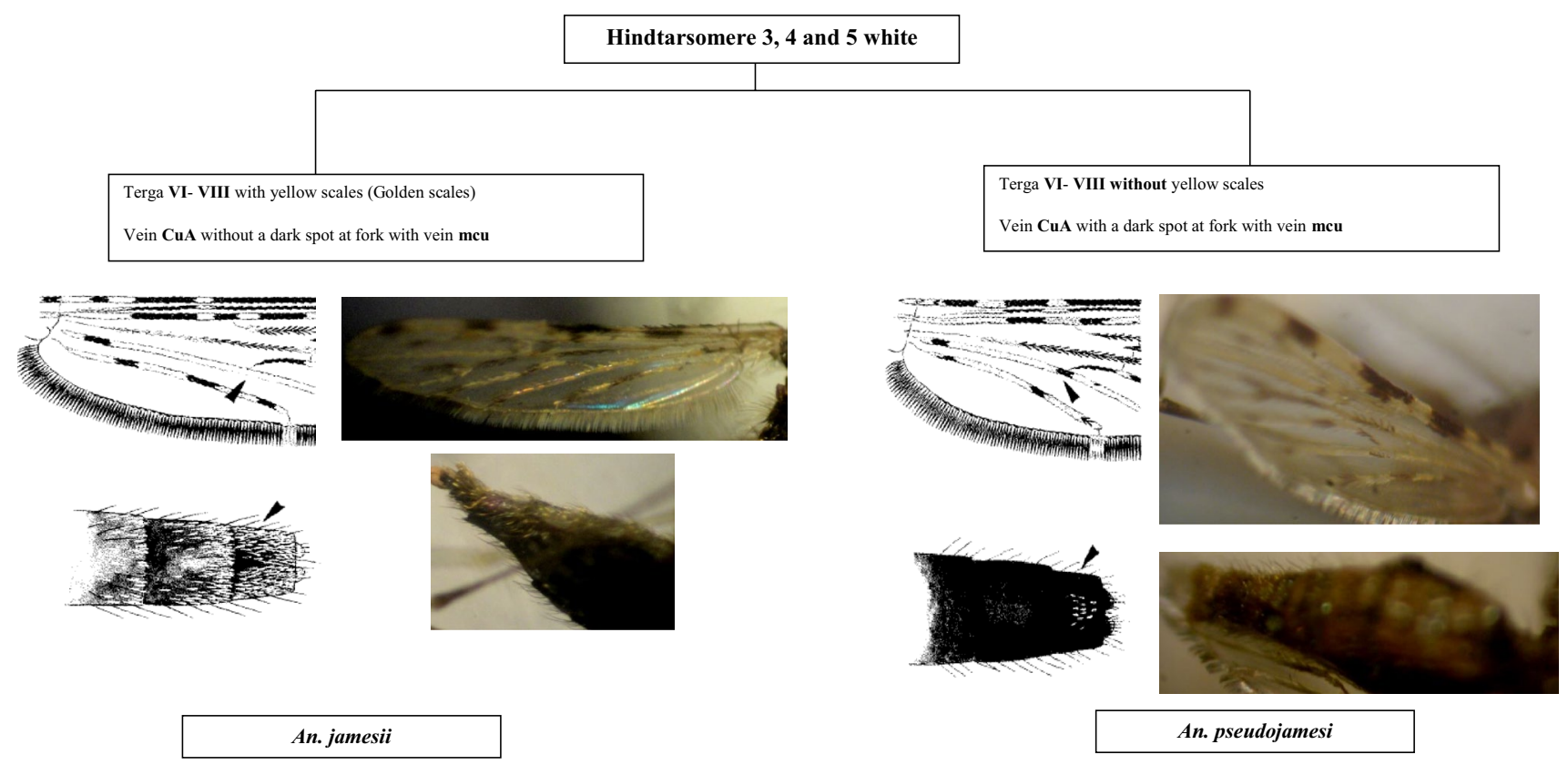

Fig. 7 Mosquitoes under Jamesii group

The pale scaling on the remigium is particularly prominent in Sri Lankan An. peditaeniatus (Amarasinghe 1990). Most specimens have a silvery white appearance (Reid 1953).
Also the mediocubital crossvein (mcu) is entirely dark scaled. Anopheles nigerrimus seems to be a dark species when compared with An. peditaeniatus. It has dark scales 


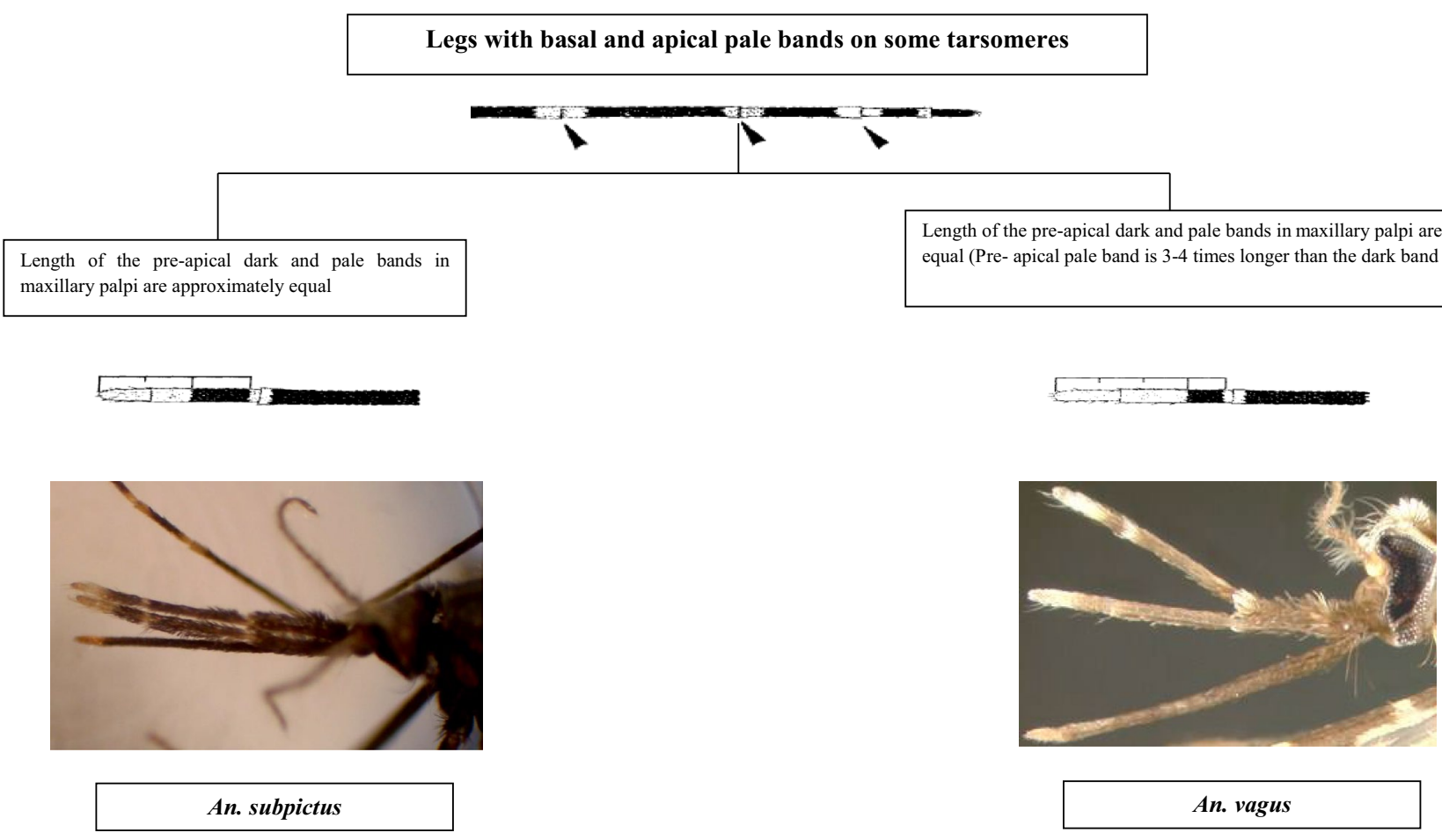

Fig. 8 Anophelines under Pyretophorous series

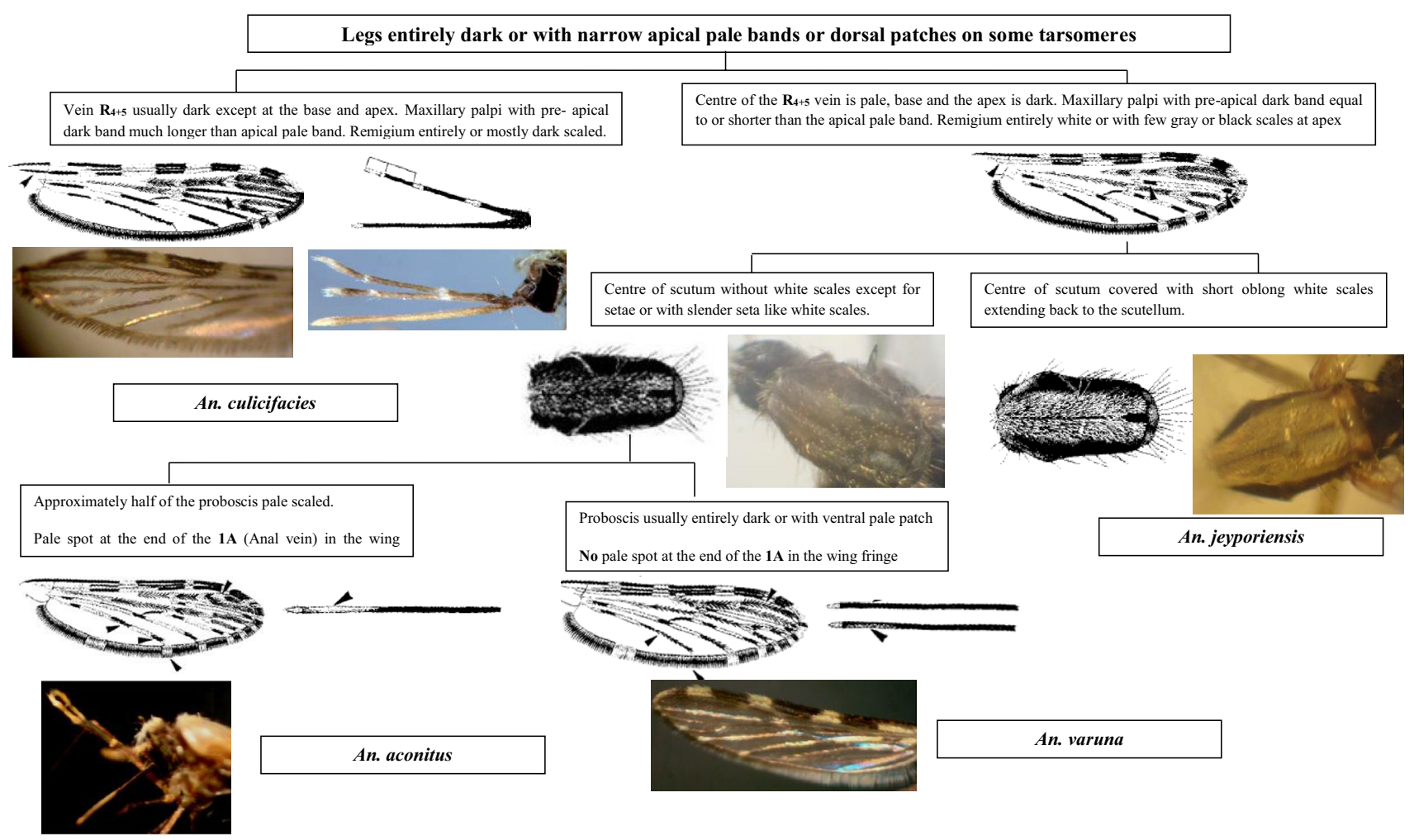

Fig. 9 Anopheles species under Myzomyia series 
on the humeral crossvein, the remigium has dark scales, the mcu has pale scales, and the apical dark mark on the anterior cubitus $(\mathrm{CuA})$ is short, rarely as long as the apical dark mark on the anal vein $(1 \mathrm{~A})$. The presence of a pale band between hindtarsomeres 3 and 4 is not reliable and has probably caused misidentifications by the field staff. Harrison and Scanlon (1975) recommended that the use of the hindtarsal bands as a key characteristic of An. peditaeniatus should be discontinued. However, continuing confusion regarding the identification of the two species could adversely impact on current malaria elimination programs in Sri Lanka, as $A n$. nigerrimus is considered as a potential vector for malaria transmission (Amarasinghe 1990; Gunathilaka et al. 2014, 2016) whereas An. peditaeniatus is not a vector.

Members of the Barbirostris group are another difficult group with regard to the separation of adults on the basis of morphological features. Of the three species present in Sri Lanka, An. reidi can be distinguished from An. barbirostris and An. barbumbrosus by the presence of speckled legs, a pale basal band on hindtarsomere 2, and pale bands crossing all four of the hind tarsal joints (Harrison, 1973).

Adults of both An. barbirostris and An. barbumbrosus have characteristics that differ from the species from Southeast Asia, but these differences apparently reflect intraspecific geographical variations. Adult An. barbirostris in the country typically have dark wings with a narrow apical fringe spot at the vein $\mathrm{R}_{4+5}$ and numerous pale scales on the abdominal sterna, characteristics that are usually found on An. campestris Reid, in Malaysia and Thailand. According to some studies conducted by Amarasinghe (1990) in the North Central and Eastern Provinces in Sri Lanka have found some An. barbirostris specimens with similar morphological features to An. campestris. However, the present study did not find any species with similar morphological features to An. campestris.

Some recent studies conducted in Sri Lanka has characterized An. barbirostris using mtDNA cytochrome oxidase subunit I (COI) and ribosomal RNA internal transcribed spacer 2 (ITS2) gene sequences. These studies have assumed that the specimens collected from widely separated locations in Sri Lanka with morphology characteristics of An. barbirostris s.l. form a new molecular type with close resemblance to An. barbirostris s.s from Indonesia and Thailand (Gajapathy et al. 2014).

Adult An. barbumbrosus in Sri Lanka has the wing with an accessory apical pale fringe spot at vein $\mathrm{R}_{4+5}$ to $\mathrm{M}_{1+2}$ and abdominal sterna without pale scales which are similar in morphological features to An. reidi. Adults of the Barbirostris group obtained during field collections always suffer varying degrees of damage to the wing fringe. Hence, the identification on the basis of the fringe spots is difficult or impossible. Therefore, the abdominal and limb characteristics are thus the most workable for routine basis.
Species of the Lophoscelomyia series can be distinguished from the other Sri Lankan members of subgenus Anopheles by the following combination of characteristics: (i) wing with dark and pale scales; (ii) antepronotum with scales; (iii) apex of hind femur with prominent tufts of erect black and white scales; (iv) head with erect broad scales on vertex; (v) scutum pale centrally and dark laterally, pleura dark; (vi) coxae pale, fore and mid coxae with long, curved scales projecting posterior-ventrally; (vii) tarsomeres without pale bands; and (viii) abdominal sternum VIII with scales (Harrison and Scanlon, 1975). A single species, An. interruptus, belonging to the Asiaticus group is present in Sri Lanka.

The Anopheles series is considered as an extremely variable series and thus difficult to define; most species in this series cannot be reliably separated on the basis of adult female morphology (Amarasinghe 1990). There are three recorded species (An. aitkenii, An. peytoni, and An. gigas) in Sri Lanka. Anopheles aitkenii can be separated from others by the absence of scales in the antepronotal lobe; long and narrow erect head scales, slightly expanded apically.

Anopheles peytoni is a unique and well-differentiated species that exhibits characteristics similar to several diverse members of the Aitkenii group. Generally, the adult females are mostly similar to aitkenii, bengalensis, borneensis, fragilis, stricklandi, and tigertti (Kulasekara et al. 1988). Most of the other members of the Aitkenii group have culicine like in general. The antennae of male $A n$. peytoni are strongly plumose like female antennae. Otherwise, adult An. peytoni cannot be separated from the other Aitkenii group members except by male genitalia characteristics.

Anopheles gigas has narrowly banded palp and tarsi and a few scales on the coxae (Amarasinghe 1990; Reid 1968). The wing is brightly ornamented. It can be easily mistaken for that of subgenus Cellia, but there is no accessory sector pale (ASP) spot in costa and vein $\mathrm{R}-\mathrm{R}_{1}$ lacks four dark spots, which are specific for the subgenus Cellia. However, this species can be separated by the presence of dark wing fringe except a pale patch between $\mathrm{CuA}$.

The following morphological features distinguish species of this subgenus from those of subgenus Anopheles: wing with four or more dark marks involving both costa and veins $\mathrm{R}-\mathrm{R}_{1}$, presence of ASP spot on cosa and/or subcosta. The Sri Lankan anophelines of this subgenus belong to four taxonomic series: the Myzomyia, Pyretophorous, Neocellia, and Neomyzomyia series.

Under the Myzomyia series there are four species in Sri Lanka (An. aconitus, An. culicifacies, An. jeyporiensis, and An. varuna). All these four species have common morphological characteristics such as propleuron with 1-4 setae, palpi with three pale bands, antepronotum without scales, legs entirely dark with narrow apical pale bands on some 
tarsomeres and abdominal segments VII-VIII, and female cerci without scales (Amarasinghe 1990; Reid 1968).

Anopheles aconitus can be recognized from other members especially by the presence of a flavescence proboscis, extensive pale scalation of wing vein $\mathrm{R}_{4+5}$, and wide pale apical and subapical palpal bands and pale spot at the end of the $1 \mathrm{~A}$ in the wing fringe can be considered as secondary identification features.

Anopheles culicifacies has maxillary palpi with a preapical dark band much longer than the apical pale band, remigium entirely or mostly dark scaled, and vein $\mathrm{R}_{4+5}$ usually dark except at the base and apex.

The following characteristics are common for both $A n$. varuna and An. jeyporiensis: (i) apical $1 / 2$ of the proboscis is not white; (ii) no pale spot at the end of $1 \mathrm{~A}$ in the wing fringe; and (iii) center of $\mathrm{R}_{4+5}$ vein is pale except the base and apex. Anopheles jeyporiensis is a unique and welldifferentiated species that exhibits characteristics similar to several diverse members of the Myzomyia series. However, they can be separated from others by the center of the scutum with pale scales extending to scutellum and vein R1 with an accessory pale spot on the preapical dark (PD) area (Gunathilaka et al. 2013, 2015; Gunathilaka 2014). Anyhow, previous studies confirmed that this species is rather variable, especially in the wing markings. The proportion of dark and pale on the female palp is also rather variable (Christophers 1933).

Pyretophorous series can be separated from the other series of subgenus Cellia in Sri Lanka by the following combination of characteristics: (i) propleuron with 1-4 setae; (ii) hind tarsomere 5 at least partially dark; (iii) palps with three pale bands; (iv) antepronotum without scales; (v) fore leg with basal and apical pale bands on some tarsomeres; and (vi) abdominal segments vi-viii and female cerci with at least a few scales (Harrison 1980; Reid 1968). Two species, An. subpictus and An. vagus, occur in Sri Lanka. Apart from the key characteristic of the palpal banding pattern, there are no other reliable features for separating these two species, except possibly for the pale patch at the apex of the proboscis in An. vagus, which is absent in An. subpictus (Reid 1968). However, samples collected from coastal side of the Vankalai area in the Mannar District of Sri Lanka presented some morphological features similar to An. sundicus, An. pseudosundicus, and An. epiropticus (Gunathilaka et al. 2011). Therefore, more studies are essential to explore this context with the aid of molecular entomological tools.

There are six species in Sri Lanka under the Neocellia series (Annularis group: An. annularis, An. pallidus; Jamesii group: An. jamesii, An. pseudojamesi; An. maculatus and An. karwari). The following features can be listed as unique features for this series: (i) absence of propleural setae; (ii) hindtarsomere 5 entirely pale scaled; and (iii) mesonotum with broad pale or white scales (Amarasinghe 1990; Reid 1968). For easy identification these six species can be categorized as An. jamesii, An. pseudojamesi, and An. maculatus having speckled legs and An. annularis, An. pallidus, and An. karwari having no speckled legs. Of these, An. karwari and An. maculatus have only the last hindtarsi entirely white. However, these two species can be separated by the presence of speckled legs only in An. maculatus. Other members have hindtarsi 3-5 entirely white. From these An. annularis and An. pallidus can be separated by the absence of speckled legs.

The wing of An. annularis is extensively dark scaled and the vein $\mathrm{CuA}$ is mostly dark scaled, with a dark spot at the fork with the vein mcu, whereas the wing of An. pallidus is pale in color and the vein $\mathrm{CuA}$ mostly pale scaled, without a dark spot at the fork with the vein mcu. Furthermore, $A n$. annularis has a white band on the apical 1/4 or less in the hindtarsomere 2, whereas An. pallidus has a white band on apical $1 / 3$ or $1 / 2$ of the hindtarsomere 2 . However, the ratio of white area on the hindtarsomere 2 is variable in both species and difficult to use as the man point of differentiation.

Separation of An. jamesii can be done by the presence of golden color scales in the abdominal terga vi-viii and vein $\mathrm{CuA}$ without a dark spot at the fork with vein mcu, whereas An. pseudojamesi has no golden color scales in the abdominal terga vi-viii and vein $\mathrm{CuA}$ with a dark spot at the fork with the vein mcu.

The Neomyzomyia series includes two species, An. tessellatus and An. elegans. The wings of these two species are highly spotted on vein radius (R), media (M), $\mathrm{CuA}$, and 1A. Commonly they have propleuron with $1-4$ setae; antepronotum with scales; palps with four or more pale bands; hindtarsomere 5 at least partly dark scaled; legs usually speckled with pale patches; and wings with many small dark marks, usually four or more in vein 1A. (Harrison 1980; Reid 1968). In general the presence of four or more spots on vein $1 \mathrm{~A}$ is useful to distinguish these two species from other Sri Lankan anophelines.

Anopheles tessellatus has a flavescence proboscis like in An. aconitus. This similarity means that there is a considerable chance of misidentifying the species as An. aconitus. Therefore, the species should be tested for speckled leg characteristics and number of wing spots for accurate identification. Anopheles elegans has a unique feature to distinguish it from others. It has a large white band crossing the tibia-tarsal joint of the hind leg.

Morphological identification keys prepared with digital pictures may facilitate accurate identification by field taxonomists. Key diagnostic characteristics have been highlighted with diagrams and digital photographs in order to help taxonomists recognize distinguishable morphological characteristics. This key is meant as an aid to the rapid identification of anophelines in Sri Lanka. It has thus been 
made as simple and concise as possible, using a few recognized primary characteristics at each step. Steps that would assign various species to their respective series and species groups are included wherever essential. Each characteristic has been described through a technical description. Hence, this study would be essential for speedy and accurate species identification in order to strengthen and support current malaria elimination programs through appropriate vector identification.

\section{Conclusion}

The identification of adult anopheline mosquitoes is an important aspect of malaria surveillance and control programs.

Acknowledgements This research work was supported by the Global Fund for Aids, Tuberculosis and Malaria (GFATM-Round 8-SRL809G11M) through Malaria Elimination Program of Tropical and Environmental Diseases and Health Associates (TEDHA) Pvt. Ltd.

Open Access This article is distributed under the terms of the Creative Commons Attribution 4.0 International License (http://creativecommons.org/licenses/by/4.0/), which permits unrestricted use, distribution, and reproduction in any medium, provided you give appropriate credit to the original author(s) and the source, provide a link to the Creative Commons license, and indicate if changes were made.

\section{References}

Amarasinghe FP (1990) A guide to the identification of anopheline mosquitoes (Diptera; Culicidae) of Sri Lanka. I Adult females. Ceylon J Sci 21:1-16

Carter HF (1950) Ceylon mosquitoes list species and names of mosquitoes recorded from Ceylon. Ceylon J Sci 24:85-115

Christophers SR (1933) The fauna of British India, including Ceylon and Burma. Diptera. In: Family Culicidae, Tribe Anophelini, vol 4. Taylor \& Francis, London, pp 371-372

Colless DH (1957) Further notes on the systematic of the Anopheles leucosphyrus group (Diptera: Culicidae). Proc R Entomol Soc Lond 26:9-131

Gajapathy K, Jude PJ, Goodacre SL, Peiris LBS, Ramasamy R, Surendran SN (2014) Molecular characterization of the malaria vector Anopheles barbirostris van der Wulp in Sri Lanka. Parasit Vectors. 7:348

Gunathilaka PADHN (2014) Distribution of major and potential malaria vectors in Mannar and Trincomalee districts and systematics of anophelines in Sri Lanka. Ph.D. Thesis, University of Kelaniya, Sri Lanka

Gunathilaka PADHN, Fernando MAST, Premasiri DS, Hapugoda MD, Wijeyerathne P, Wickramasinghe AR, Abeyewickreme W (2011) Morphological differences among Anopheles subpictus sibling species B breeding in wastewater habitats in Mannar District, Sri Lanka. Annual Research Symposium, University of Kelaniya, Sri Lanka, 12, p 50
Gunathilaka PADHN, Fernando MAST, Hapugoda MD, Wijeyerathne P, Wickramasinghe AR, Abeyewickreme W (2013) Appearance of Anopheles jeyporiensis (James) in Sri Lanka after 106 years. In: Proceedings of the annual session of Sri Lanka Association of Advancement Sciences, Colombo, Sri Lanka, 69, p 202

Gunathilaka N, Fernando T, Hapugoda M, Wickremasinghe R, Abeyewickreme W (2014) Revised morphological identification key to the larval anopheline (Diptera: Culicidae) of Sri Lanka. Asian Pac J Trop Biomed 3(12):930-935

Gunathilaka N, Hapugoda M, Wickremasinghe R, Abeyewickreme W (2015) Appearance of Anopheles jeyporiensis James from Sri Lanka; a new species to the mosquito checklist. Med Entomol Zool 66(3): 1-5

Gunathilaka N, Denipitiya T, Hapugoda M, Abeyewickreme W, Wickremasinghe $\mathrm{R}$ (2016) Determination of the foraging behaviour and blood meal source of malaria vector mosquitoes in Trincomalee District of Sri Lanka using a multiplex real time polymerase chain reaction assay. Malar J 15:242

Harbach RE, Knight KL (1980) Taxonomists' glossary of mosquito anatomy. Plexus, Marlton, p 415

Harbach RE, Knight KL (1982) Corrections and additions to taxonomists' glossary of mosquito anatomy. Mosq Syst 13:17-201

Harrison BA (1973) Anopheles (An.) reidi, a new species of barbirostris species complex from Sri Lanka (Diptera: Culicidae). Proc Entomol Soc Wash 75(3):365-371

Harrison BA (1980) Medical entomology studies-XIII. The Myzomyia series of Anopheles (Cellia). In Thiland, with emphasis on intra-interspecific variations (Diptera: Culicidae). Contrib Am Entomol Inst 17(4):1-195

Harrison BA, Scanlon JE (1975) The subgenus Anopheles in Thailand (Diptera: Culicidae). Contrib Am Entomol Inst (Ann Arbor) 12(1):1-307

Jayasekara N, Chelliah RV (1981) An annoted checklist of mosquitoes of mosquitoes of Sri Lanka. MAB-UNESCO-Man and the Biosphere National Committee for Sri Lanka, 8, pp 1-16

Knight KL, Stone A (1977) A catalog of the mosquitoes of the world (Diptera: Culicidae), 2nd edn. Entomology Society of America, Maryland, pp 1-611

Kulasekara VL, Harrison BA, Amerasinghe FP (1988) Anopheles (Anopheles) peytoni n.sp, the "An. insulaeflorum" auct. From Sri Lanka (Diptera: Culicidae). Mosq Syst 20(3):302-316

Ramachandra RT (1984) The Anopheline of India (revised edition). Indian Council of Medical Research, New-Delhi, pp 23-72

Rattanarithikul R, Harrison BA, Harbach RE, Panthusiri P, Coleman RE (2006) Illustrated keys to the mosquitoes of Thailand IV Anopheles. Southeast Asian J Trop Med Public Health 37(Suppl 2): $1-128$

Reid JA (1953) The Anopheles hyrcanus group in South-East Asia (Diptera: Culicidae). Bull Entomol Res 44:5-76

Reid JA (1968) Anopheles mosquitoes of Malaya and Borneo. Inst Med Res Malays 44:1-520

Reinert JF (1975) Mosquito generic and subgeneric abbreviations (Diptera: Culicidae). Mosq Syst 7:105-110

Reinert JF (2001) Revised list of abbreviations for genera and subgenera of Culicidae (Diptera) and notes on generic and subgeneric changes. J Am Mosq Control Assoc 17:5-51

Sallum MAM, Peyton EL, Wilkerson RC (2005) Six new species of the Anopheles leucosphyrus group, reinterpretation of Anopheles elegans and vector implications. J Med Vet Entomol 19:99-158

Wilkerson RC, Peyton EL (1990) Standardized nomenclature for the costal wing spots of the genus Anopheles and other spotted wing mosquitoes (Diptera: Culicidae). J Med Entomol 27:24-207 